\title{
Relação entre percepção sobre serviços odontológicos e condições de Saúde Bucal em hipertensos e diabéticos
}

\author{
Relationship between the perception of dental care \\ and Oral Health conditions in hypertensive and diabetic patients
}

Eduardo José Pereira Oliveira ${ }^{1}$

Denismar Alves Nogueira ${ }^{2}$

Alessandro Aparecido Pereira ${ }^{3}$

${ }^{1}$ Programa de PósGraduação em Ciências

Odontológicas,

Universidade Federal de

Alfenas (Unifal). R. Gabriel

Monteiro da Silva 700,

Centro. 37130-001 Alfenas

MG Brasil. eduardo.

oliveira@oi.com.br

${ }^{2}$ Instituto de Ciências

Exatas, Unifal. Alfenas MG

Brasil.

${ }^{3}$ Departamento de Clínica

e Cirurgia, Unifal. Alfenas

MG Brasil.

\begin{abstract}
The aim of this study was to evaluate the relationship between the perception of dental care services and oral health conditions in a hypertensive and diabetic population subscribed to the Family Health Strategy in the city of Alfenas, Minas Gerais, Brazil. This was a domiciliary, descriptive-analytical study with random and stratified sample, consisting of 186 individuals. The following indexes were applied: Decayed, Missing and Filled Teeth (DMFT); Filled and Sound Teeth (FS-T); Dental Care Index (DCI); Evaluation of the use and need of dentures; Oral health for primary care assessment questionnaire (OHPCA). A predominance of women (67.74\%), hypertensive non-diabetic people (58.60\%) and elderly people $(52.69 \%)$, with an average age of $64.26( \pm 12.22)$ years, was observed. We noticed a total DMFT of 27.00 ( \pm 6.24); FS-T = 8.94 ( \pm 10.28$) ; D C I$ $=19.42$ ( \pm 26.80$) ; 39.78 \%$ of partial dentures usage, $56.45 \%$ of total prosthesis usage with necessity of $36.02 \%$ of partial dentures and $28.49 \%$ of total prosthesis. In the OHPCA questionnaire, positive assessments of services were prevalent. Oral Health proved to be best among those individuals who reported good relationship with their dentists and worse among those individuals who considered as good the equipment used in the services. The critical aspects were: access, speed and professional-patient communication.
\end{abstract}

Key words Health evaluation, Dental health services, Oral health
Resumo O objetivo deste estudo foi avaliar a relação entre a percepção sobre os serviços odontológicos e a saúde bucal numa população de hipertensos e diabéticos adscritos à Estratégia Saúde da Família em Alfenas, MG, Brasil. Estudo domiciliar, descritivo-analítico, com amostra randomizada e estratificada, composta por 186 sujeitos. Aplicaram-se os indices: Dentes Cariados, Perdidos e Obturados (CPOD); Índice de Dentes Funcionais (FS-T); Índice de Cuidados Odontológicos (ICO); Avaliação do uso e necessidade de próteses; Questionário de Avaliação da Saúde Bucal na Atenção Primária (ASBAP-usuário). Observouse predominio de mulheres $(67,74 \%)$, hipertensos não diabéticos (58,60\%) e idosos (52,69\%) - idade média de 64,26 $( \pm 12,22)$ anos. Registrou-se $C P O D=27,00( \pm 6,24) ; F S-T=8,94( \pm 10,28)$; $I C O=19,42( \pm 26,80) ; 39,78 \%$ de uso de próteses parciais, $56,45 \%$ de próteses totais e necessidades de 36,02\% de próteses parciais e 28,49\% de próteses totais. No ASBAP-usuário, prevaleceram avaliações positivas dos serviços. A Saúde Bucal mostrou-se melhor entre os sujeitos que relataram bom vínculo com o dentista e pior entre os que consideraram bons os equipamentos utilizados nos Serviços, sendo ainda pontos críticos: acesso, rapidez e comunicação profissional-paciente.

Palavras-chave Avaliação em saúde, Serviços de saúde bucal, Saúde bucal 


\section{Introdução}

Segundo Donabedian, a 'avaliação de serviços de saúde' consiste em um processo sistemático e objetivo que busca analisar a efetividade ou o impacto de determinada atividade, a partir de objetivos predeterminados, com vistas à reorientação em prol da conquista de benefícios ${ }^{1}$. A visão do usuário como protagonista nesse processo torna-se importante. A expressão de suas experiências singulares quando submetidos ao cuidado permite um julgamento mais adequado sobre as características e qualidade dos serviços ofertados ${ }^{2,3}$. Além disso, a expressão de sua visão reafirma direitos individuais e de cidadania, contribuindo para o empoderamento e expressando uma dimensão política e social ${ }^{4}$.

Vários são os instrumentos que visam avaliar os serviços de saúde, agregando visões de diferentes atores (gestores, profissionais de saúde e usuários), sob perspectivas diversas - que vão da estruturação do serviço ao grau de satisfação dos usuários ${ }^{5,6}$. No Brasil, para a Avaliação de Serviços Odontológicos, observa-se uma incipiente consolidação de instrumentos elaborados ou adaptados à realidade local, como o QASSaB (Questionário de Avaliação da Qualidade dos Serviços de Saúde Bucal) $)^{7}$, o PCATool-Brasil SB (Instrumento de Avaliação da Atenção Primária-Brasil, adaptado à Saúde Bucal $)^{5}$ e o ASBAP -usuário (Avaliação da Saúde Bucal na Atenção Primária versão usuário $)^{8}$.

Apesar de percepção e satisfação tratarem do mesmo objeto, faz-se necessário diferenciá-las. A percepção exprime um julgamento pessoal a respeito de uma pauta ${ }^{9}$. A satisfação, porém, só pode ser verificada quando uma necessidade coexiste a ela. Assim, usuários são capazes de avaliar um serviço quando, em algum momento, demandam por ele ${ }^{10}$. O conhecimento acerca da satisfação, anseios e necessidades dos usuários dos serviços atrelados à identificação dos determinantes dessas percepções podem se mostrar importantes ferramentas no caminho da reorientação de práticas e modelos assistenciais. Há de se ponderar, por outro lado, que o grau de satisfação dos usuários pode apresentar-se elevado mesmo diante de baixo desempenho dos serviços ${ }^{11}$.

Entretanto, a incorporação de variáveis descritoras de Condições Clínicas de Saúde Bucal (CCSB) à investigação das percepções de usuários sobre serviços odontológicos, mostra-se bastante oportuna, pois essas refletem o objeto e objetivo final dos serviços, sendo, em maior ou menor grau, uma medida da efetividade de ações e programas.
Com o decurso das transições demográfica e epidemiológica, o significativo incremento de Doenças Crônicas Não Transmissíveis (DCNT) tem gerado a necessidade de políticas públicas específicas às novas demandas ${ }^{12,13}$. Nesse contexto, destacam-se os portadores de Hipertensão Arterial Sistêmica (HAS), Diabetes Mellitus (DM) e a comprovada relação bidirecional entre saúde bucal e sistêmica nesses quadros ${ }^{14-16}$.

A HAS e o DM atingem predominantemente adultos e idosos. E nesses ciclos de vida é mais comum se observar as consequências da cárie dentária (edentulismo e uso/necessidade de próteses) que sua atividade propriamente dita. De maneira geral, observa-se maior prevalência de cáries entre crianças e adolescentes, diminuindose sua incidência na idade adulta, quando surgem com maior severidade alterações periodontais (gengivites e destruições ósseas), que conduzem à perda dental ${ }^{17}$. Desse modo, são pertinentes investigações que incorporem medidas da extensão da cárie e suas consequências - como o Somatório de Dentes Cariados, Perdidos e Obturados $(\mathrm{CPOD})^{18}$; bem como informações sobre a presença de dentição previsivelmente funcional - assim como o Índice de Dentes Funcionais (FST) ${ }^{19}$; dimensões que caracterizem a orientação dos Serviços Odontológicos - tal qual se propõe no Índice de Cuidados Odontológicos (ICO ${ }^{20}$; e medidas sobre os produtos dos serviços (uso/ necessidade de próteses $)^{21}$.

Dessa forma, o presente estudo teve como objetivo avaliar a relação entre a percepção sobre os serviços odontológicos e condições clínicas de saúde bucal numa população de adultos e idosos hipertensos e diabéticos.

\section{Metodologia}

Este trabalho integra uma pesquisa descritivo-analítica, transversal, epidemiológica, domiciliar, onde se avaliou além da percepção dos usuários acerca dos serviços odontológicos e CCSB, a qualidade de vida relacionada a essas variáveis entre adultos e idosos hipertensos e diabéticos vinculados à Estratégia Saúde da Família (ESF) em Alfenas, sul do Estado de Minas Gerais, Brasil. O município está situado a $342 \mathrm{~km}$ da capital Belo Horizonte, tendo sua população estimada, em 2014, em 78.176 habitantes. O estudo foi aprovado pelo Comitê de Ética em Pesquisa com Seres Humanos da Universidade Federal de Alfenas e Secretaria Municipal de Saúde de Alfenas/MG.

Determinou-se, inicialmente, o tamanho mínimo da amostra a partir de critérios pertinentes 
aos levantamentos em saúde bucal, adotando-se para o cálculo uma fórmula para populações finitas proposta por Silva ${ }^{22}$. Como referência, considerou-se o CPOD registrado no levantamento nacional em saúde bucal SB BRASIL $2010^{23}$ para sujeitos em idades entre 35-44 e 65-74 anos, residentes em cidades do interior do Sudeste Brasileiro. Adotou-se nível de confiança de 95\%, erro de $10 \%$, recurso deff (design effect) (em que se dobra o valor obtido inicialmente) e taxa de não resposta de $20 \%$, obtendo-se amostra final mínima de 216 sujeitos para a investigação das CCSB. Porém, por se tratar de um estudo domiciliar, onde a busca ativa é a única ferramenta de captação e considerando-se as possibilidades de recusa e dificuldades na localização dos potenciais participantes, decidiu-se organizar um cadastro de reserva com o dobro de sujeitos elegíveis.

Para sorteio dos sujeitos, incialmente definiuse que cinco entre as 15 ESF presentes na zona urbana do município deveriam compor amostra, sorteando-as. Na sequência, realizou-se um levantamento dos indivíduos cadastrados no sistema HIPERDIA/SUS (Sistema de Cadastramento e Acompanhamento de Hipertensos e Diabéticos do Sistema Único de Saúde) nas ESF eleitas, registrando-se um total de 2629 sujeitos. Para a seleção dos possíveis participantes, enumerou-se os indivíduos em uma planilha, listando-os de acordo com a ESF, numa sequência de microáreas, ruas e números crescentes dos domicílios. $\mathrm{O}$ sorteio sistematizou a amostra através de um intervalo regular obtido pela divisão da população total (2.629) pela amostra dobrada - referente ao cadastro de reserva - (432), obtendo-se um intervalo igual a 6,1. O primeiro indivíduo foi sorteado por uma tabela de números aleatórios de 1 a 6. Sendo o número 6 o escolhido, o primeiro integrante foi o sexto da lista. A partir daí, a cada sujeito sorteado, somou-se o intervalo de 6,1, procedendo-se aos arredondamentos necessários. Dessa forma, automaticamente, cada estrato (ESF) compôs proporcionalmente a listagem de 432 sujeitos (cadastro de reserva).

Para ser incluído na amostra, o sujeito deveria ser portador de HAS e/ou DM; ter mais de 35 anos; estar vinculado a uma das ESF selecionadas e ter se submetido a pelo menos duas consultas com alguma Equipe de Saúde Bucal (ESB). Como ESB, consideraram-se quaisquer arranjos entre profissionais que envolvesse um cirurgiãodentista na prestação de serviços odontológicos. Dessa forma, foram estabelecidas três categorias de serviços: ESB integrante de ESF; Clínicas de Ensino de duas universidades do município - uma pública outra privada; Clínicas/consultórios privados. Consideraram-se 'idosos', os sujeitos com mais de 65 anos, de acordo com as recomendações da Organização Mundial de Saúde para levantamentos epidemiológicos em Saúde Bucal $^{18}$.

Para a avaliação das percepções dos sujeitos sobre os serviços odontológicos, utilizou-se o questionário ASBAP-usuário ${ }^{8}$, na modalidade autopreenchimento, em versão modificada. O instrumento é composto por cindo dimensões: Acolhimento (questões 3, 6, 8, 9, 10, 17, 18); Qualidade da atenção (questões 4, 5, 7,12, 14, 15, 19); Acesso (questões 1, 11, 20); Equipe (questões 13, 16); Organização (questão 2). Escolheu-se tal instrumento por ter sido esse desenvolvido também com populações de adultos e idosos no âmbito da ESF. Entretanto, para adaptação às especificidades desta investigação, procedeu-se a algumas modificações. Considerando-se a possibilidade de utilização de serviços odontológicos que não fossem a ESF, decidiu-se suprimir as questões 13, 15,16 e 19 - por referirem-se a aspectos específicos da ESF. Solicitou-se ao respondente que substituísse a palavra "posto" por "consultório ou clínica”. E, por fim, manteve-se a escala original no momento da aplicação dos questionários. Porém, durante a análise dos dados, decidiu-se excluir as respostas assinaladas com a opção "Não sei" (Quadro 1).

As CCSB foram investigadas através dos índices CPOD (segundo os códigos e critérios preconizados pela OMS) ${ }^{18}$; FS-T (soma de dentes hígidos e restaurados - presta-se a verificar o número de dentes previsivelmente funcionais na cavidade oral, sendo que quão mais próximo de 32 se situar o índice, maior é o número de dentes funcionais) ${ }^{19}$; e ICO (quociente entre o número de dentes restaurados e o somatório de cariados, perdidos e restaurados, multiplicado por 100 - expressa a capacidade do serviço em atender as necessidades de tratamento restaurador $)^{20}$. Avaliou-se também o uso e necessidade de próteses por modalidade (próteses parciais e totais), segundo os códigos e critérios adotados no SB BRASIL $2010^{21}$. Os exames foram realizados nas residências dos sujeitos, sob luz natural, com auxílio da sonda ballpoint (OMS) e espelho bucal plano, estando os sujeitos posicionados em decúbito dorsal, com o examinador em 12 horas $^{18}$.

Os dados foram coletados por um examinador devidamente treinado e calibrado. Realizouse o treinamento e a calibração do examinador em duas ESF com sujeitos que não compuseram a amostra final. Participaram dessa etapa 36 vo- 
Quadro 1. Questionário ASBAP-usuário de Sanchez

\begin{tabular}{|c|c|c|c|c|}
\hline 1 & $\begin{array}{l}\text { É fácil conseguir tratamento odontológico no posto onde sou } \\
\text { atendido. }\end{array}$ & Sim / às vezes & Não / nunca & Não sei \\
\hline 2 & $\begin{array}{l}\text { Para consultar com o dentista que me atende nesse posto, eu } \\
\text { preciso deixar de trabalhar por um período do dia }\end{array}$ & Sim / às vezes & Não / nunca & Não sei \\
\hline 3 & $\begin{array}{l}\text { Conversar com o dentista que me atende no posto sempre que eu } \\
\text { preciso é fácil. }\end{array}$ & Sim / às vezes & Não / nunca & Não sei \\
\hline 4 & O dentista que me atende no posto faz até mais do que eu preciso. & Sim / às vezes & Não / nunca & Não sei \\
\hline 5 & $\begin{array}{l}\text { Eu recomendo o posto de saúde onde faço tratamento } \\
\text { odontológico para outras pessoas. }\end{array}$ & Sim / às vezes & Não / nunca & Não sei \\
\hline 6 & O dentista que me atende no posto me escuta com atenção. & Sim / às vezes & Não / nunca & Não sei \\
\hline 7 & Eu entendo bem aquilo que o dentista do posto me explica. & Sim / às vezes & Não / nunca & Não sei \\
\hline 8 & O dentista que me atende no posto me conhece bem. & Sim / às vezes & Não / nunca & Não sei \\
\hline 9 & O dentista que me atende no posto se relaciona bem comigo. & Sim / às vezes & Não / nunca & Não sei \\
\hline 10 & $\begin{array}{l}\text { O dentista que me atende no posto se preocupa com minha } \\
\text { saúde. }\end{array}$ & Sim / às vezes & Não / nunca & Não sei \\
\hline 11 & $\begin{array}{l}\text { No posto onde sou atendido me orientam para fazer consulta de } \\
\text { retorno com o dentista que me atende. }\end{array}$ & Sim / às vezes & Não / nunca & Não sei \\
\hline 12 & $\begin{array}{l}\text { Tenho confiança nos trabalhos que o dentista que me atende no } \\
\text { posto faz. }\end{array}$ & Sim / às vezes & Não / nunca & Não sei \\
\hline $13^{*}$ & $\begin{array}{l}\text { Quando outros profissionais da saúde ajudam o dentista que me } \\
\text { atende no posto o resultado do tratamento é melhor. }\end{array}$ & Sim / às vezes & Não / nunca & Não sei \\
\hline 14 & $\begin{array}{l}\text { O trabalho do dentista que me atende no posto é de boa } \\
\text { qualidade. }\end{array}$ & Sim / às vezes & Não / nunca & Não sei \\
\hline $15^{*}$ & $\begin{array}{l}\text { Minhas necessidades de saúde bucal são resolvidas com a mesma } \\
\text { qualidade que o tratamento feito por um dentista particular. }\end{array}$ & Sim / às vezes & Não / nunca & Não sei \\
\hline $16^{*}$ & $\begin{array}{l}\text { Os agentes comunitários de saúde fazem um trabalho importante } \\
\text { para a comunidade. }\end{array}$ & Sim / às vezes & Não / nunca & Não sei \\
\hline 17 & $\begin{array}{l}\text { De maneira geral, o posto onde sou atendido é limpo e } \\
\text { organizado. }\end{array}$ & Sim / às vezes & Não / nunca & Não sei \\
\hline 18 & Os equipamentos usados são bons e funcionam bem. & Sim / às vezes & Não / nunca & Não sei \\
\hline $19^{*}$ & $\begin{array}{l}\text { O dentista que me atende no posto faz um bom trabalho, } \\
\text { independentemente do sistema político. }\end{array}$ & Sim / às vezes & Não / nunca & Não sei \\
\hline 20 & O tratamento com o dentista que me atende no posto é rápido. & Sim / às vezes & Não / nunca & Não sei \\
\hline
\end{tabular}

* Questões excluídas por referirem-se à aspectos específicos da ESF.

luntários (16 no treinamento e 20 na calibração). Tomando por referência um examinador padrão (Gold Standard), aferiu-se a estatística kappa interexaminador para o CPOD (kappa $=0,91)$, além de uso e necessidade de próteses (kappa $=1,00$ ). Para tabulação e análise dos dados, foi utilizado o pacote estatístico SPSS ${ }^{\circledast} 22.0$, adotando-se nível de significância de 5\% $(\alpha<0,05)$. Considerandose a não Normalidade na distribuição dos dados verificada pelo teste de Kolmogorov-Smirnov $(\rho<0,05)$, testes não paramétricos foram utilizados. Aplicou-se o Teste U de Mann-Whitney para comparação de médias e os testes de Quiquadrado (para frequências observadas maiores que cinco) ou Exato de Fisher (para frequências observadas menores que cinco) para a comparação de proporções.

\section{Resultados}

Submeteram-se ao levantamento das CCSB 218 sujeitos, alcançando-se a composição amostral mínima (216). Contudo, não responderam ao ASBAP-usuário: 24 sujeitos que não liam e escreviam ou apresentavam alguma dificuldade cognitiva; quatro sujeitos que relataram confeccionar suas próteses diretamente com o protético e quatro sujeitos que não foram ao dentista pelo menos nos últimos dez anos, obtendo-se na amostra final 186 respondentes (Gráfico 1).

No Tabela 1, apresentam-se dados da distribuição da amostra por sexo, ciclo de vida, condição sistêmica, além da caracterização das CCSB e da Percepção sobre os Serviços (ASBAP-usuário). Observou-se predomínio de mulheres $(67,74 \%)$, 


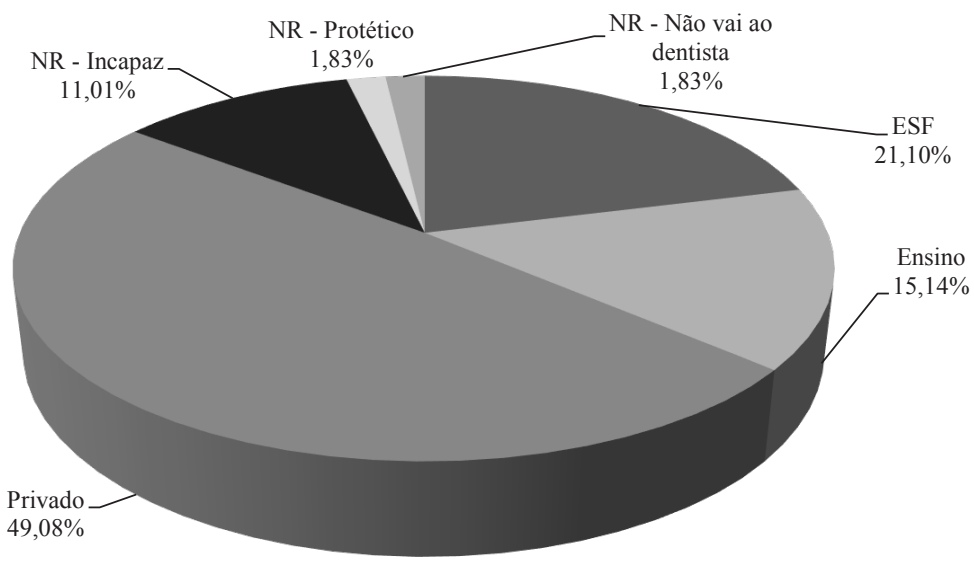

Gráfico 1. Distribuição da amostra entre respondentes (segundo a utilização de Serviços Odontológicos) e não respondentes. Alfenas, MG, Brasil, $2016(\mathrm{n}=218)$.

Legenda: NR = não respondeu ao ASBAP-usuário; Incapaz = incapaz de responder ao questionário por não ler e escrever ou apresentar alguma dificuldade cognitiva.

Tabela 1. Distribuição da amostra segundo o sexo, ciclo de vida, condição sistêmica, Condições Clínicas de Saúde Bucal e Percepção Sobre Serviços Odontológicos (ASBAP-usuário) entre adultos e idosos. Alfenas, MG, Brasil, 2016 ( $\mathrm{n}=186$ ).

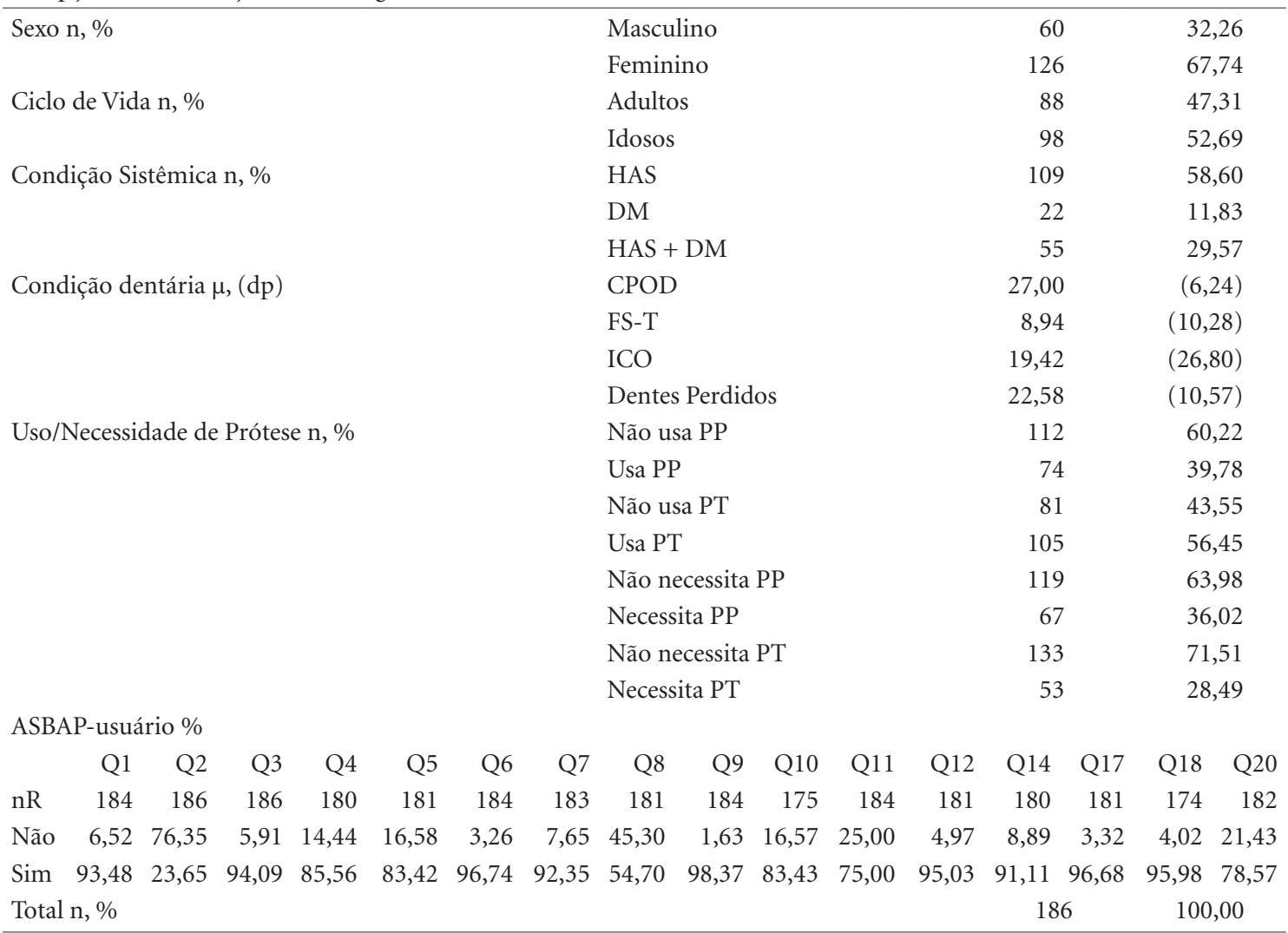

Legenda: $\mu=$ média; $(\mathrm{dp})=$ desvio padrão; HAS = Hipertensão Arterial Sistêmica; DM = Diabetes Mellitus; FS-T = Índice de Dentes Funcionais; ICO = Índice de Cuidados Odontológicos; PP = Prótese Parcial; PT = Prótese Total; $\mathrm{Q}^{\mathrm{n}}=$ Questão n; nR = número de respondentes de cada questão do ASBAP-usuário. 
hipertensos não diabéticos $(58,60 \%)$ e idosos $(52,69 \%)$. A idade média entre os 186 respondentes foi de $64,26( \pm 12,22)$, variando entre 35 e 93 anos. Registrou-se CPOD $=27,00( \pm 6,24)$; $\mathrm{FS}-\mathrm{T}=8,94( \pm 10,28) ; \mathrm{ICO}=19,42( \pm 26,80)$; $39,78 \%$ de uso de próteses parciais, $56,45 \%$ de próteses totais e necessidades de $36,02 \%$ de próteses parciais e $28,49 \%$ de próteses totais. No ASBAP-usuário, prevaleceram avaliações positivas dos serviços.

A Tabela 2 e o Quadro 2 evidenciam a relação entre a Percepção sobre os Serviços Odontológicos e as CCSB na população estudada. A Saúde Bucal mostrou-se melhor entre os sujeitos que relataram bom vínculo com o dentista e pior entre os que consideraram bons os equipamentos utilizados nos Serviços, sendo ainda pontos críti- cos: acesso, rapidez e comunicação profissionalpaciente.

\section{Discussão}

Mesmo apresentando elevado edentulismo, sendo submetida a um cuidado pouco conservador e demandando ainda por próteses, a população estudada, de maneira geral, avalia positivamente os Serviços Odontológicos. As CCSB (CPOD, FS-T, ICO, Número de Dentes Perdidos) são melhores entre os usuários que consideram que o dentista lhes conhece bem e piores entre aqueles que julgam como bons os equipamentos utilizados nos Serviços. A necessidade de próteses mostra-se um entrave em aspectos como acesso, comuni-

Quadro 2. Síntese das relações de dependência significantes das Variáveis com a Percepção dos entrevistados sobre os Serviços Odontológicos. Alfenas, MG, Brasil, 2016.

\begin{tabular}{|c|c|}
\hline $\begin{array}{l}\text { Q1. É fácil conseguir } \\
\text { tratamento odontológico } \\
\text { onde sou atendido. }\end{array}$ & $\begin{array}{l}\text { - 96,23\% dos usuários do Serviço Privado, } 95,56 \% \text { da ESF e } 81,82 \% \text { dos } \\
\text { frequentadores das Clínicas de Ensino acreditam ser fácil conseguir tratamento } \\
\text { odontológico ( } \rho=0,011) \text {. } \\
\text { - Entre os sujeitos que não necessitam de PT, } 96,30 \% \text { acham fácil conseguir } \\
\text { tratamento. Já entre os que necessitam esse percentual é de } 86,79 \%(\rho=0,016) \text {. }\end{array}$ \\
\hline $\begin{array}{l}\text { Q3. Conversar com o dentista, } \\
\text { sempre que eu preciso, é fácil. }\end{array}$ & $\begin{array}{l}\text { - 96,32\% dos sujeitos que não necessitam de PT acham fácil conversar com } \\
\text { o dentista. Entre os que necessitam, } 88,89 \% \text { compartilham dessa visão ( } \rho= \\
0,048) \text {. }\end{array}$ \\
\hline $\begin{array}{l}\text { Q7. Eu entendo bem aquilo } \\
\text { que o dentista me explica. }\end{array}$ & $\begin{array}{l}\text { - Nas Clínicas de Ensino, } 100 \% \text { dos usuários acreditam compreender bem } \\
\text { aquilo que o dentista explica. No Serviço Privado são 93,27\% e na ESF 84,78\% } \\
\text { com a mesma opinião }(\rho=0,037) \text {. }\end{array}$ \\
\hline $\begin{array}{l}\text { Q8. O dentista que me atende } \\
\text { me conhece bem. }\end{array}$ & $\begin{array}{l}\text { - 60,58\% dos usuários do Serviço Privado, } 55,56 \% \text { da ESF e } 34,38 \% \text { daqueles } \\
\text { que frequentam as Clínicas de Ensino consideram que o dentista lhes conhece } \\
\text { bem ( } \rho=0,033) \text {. } \\
\text { - Os sujeitos que consideram que o dentista lhes conhece bem apresentam: } \\
\text { menor CPOD }(26,24 \times 28,54 ; \rho=0,005) \text { e maior FS-T }(10,84 \text { x 5,84; } \rho= \\
0,001) \text {; maior ICO }(24,59 \text { x } 11,39 ; \rho=0,001) \text { e menor número de Dentes } \\
\text { Perdidos ( } 20,57 \text { x } 25,82 ; \rho=0,001) \text { quando comparados àqueles que pensam } \\
\text { que o dentista não os conhece bem. } \\
\text { - 72,73\% dos sujeitos que não usam PT e } 43,52 \% \text { dos que usam PT consideram } \\
\text { que o dentista os conhece bem ( } \rho<0,001) \text {. } \\
\text { - } 60,61 \% \text { dos sujeitos que não necessitam de PT e } 43,40 \% \text { dos que necessitam } \\
\text { de PT pensam que o dentista os conhece bem }(\rho=0,033) \text {. }\end{array}$ \\
\hline $\begin{array}{l}\text { Q10. O dentista que me } \\
\text { atende se preocupa com } \\
\text { minha saúde. }\end{array}$ & $\begin{array}{l}\text { - 86,82\% dos sujeitos que não necessitam de PT e } 74,00 \% \text { dos que necessitam } \\
\text { de PT pensam que o dentista se preocupa com sua saúde }(\rho=0,039) \text {. }\end{array}$ \\
\hline $\begin{array}{l}\text { Q18. Os equipamentos } \\
\text { usados são bons e funcionam } \\
\text { bem. }\end{array}$ & $\begin{array}{l}\text { - Aqueles que consideram que os equipamentos utilizados pelo dentista } \\
\text { são bons ou funcionem bem apresentam piores FS-T }(8,12 \times 17,00 ; \rho= \\
0,020) \text { e ICO }(17,41 \times 44,32 ; \rho=0,026) \text { e maior número de Dentes Perdidos } \\
(23,45 \times 14,75 ; \rho=0,022) \text { quando comparados aos que consideram que os } \\
\text { equipamentos não são bons e não funcionam bem. }\end{array}$ \\
\hline $\begin{array}{l}\text { Q20. O tratamento com o } \\
\text { dentista que me atende é } \\
\text { rápido. }\end{array}$ & $\begin{array}{l}\text { - 88,37\% dos usuários da ESF, } 84,91 \% \text { do Serviço Privado e } 45,46 \% \text { dos } \\
\text { frequentadores das Clínicas de Ensino consideram rápidos seus tratamentos } \\
\text { odontológicos }(\rho<0,001) \text {. }\end{array}$ \\
\hline
\end{tabular}


Tabela 2. Relação entre a Percepção sobre os Serviços Odontológicos e Condições Clínicas de Saúde Bucal nos adultos e idosos entrevistados. Alfenas, MG, Brasil, 2016 ( $\mathrm{n}=186)$.

\begin{tabular}{|c|c|c|c|c|c|c|c|c|c|c|c|c|}
\hline & Sexo & $\mathrm{CV}$ & CS & SO & CPO & FST & ICO & $\mathbf{P}$ & UPP & UPT & NPP & NPT \\
\hline & $\rho^{Q}$ & $\rho^{Q}$ & $\rho^{\mathrm{Q}}$ & $\rho^{Q}$ & $\rho^{\mathrm{M}}$ & $\rho^{M}$ & $\rho^{\mathrm{M}}$ & $\rho^{M}$ & $\rho^{Q}$ & $\rho^{\mathrm{Q}}$ & $\rho^{Q}$ & $\rho^{Q}$ \\
\hline Q1 & 0,165 & 0,849 & 0,360 & 0,011 & 0,779 & 0,734 & 0,733 & 0,726 & 0,260 & 0,888 & 0,385 & 0,016 \\
\hline Q2 & 0,059 & 0,368 & 0,637 & 0,328 & 0,387 & 0,818 & 0,852 & 0,845 & 0,399 & 0,485 & 0,150 & 0,569 \\
\hline Q3 & 0,725 & 0,924 & 0,399 & $0,685^{\mathrm{F}}$ & 0,703 & 0,598 & 0,743 & 0,560 & 0,800 & 0,639 & 0,521 & 0,048 \\
\hline Q4 & 0,714 & 0,996 & 0,948 & 0,302 & 0,978 & 0,832 & 0,697 & 0,758 & 0,491 & 0,588 & 0,305 & 0,486 \\
\hline Q5 & 0,466 & 0,820 & 0,726 & 0,427 & 0,337 & 0,398 & 0,730 & 0,472 & 0,872 & 0,762 & 0,362 & 0,280 \\
\hline Q6 & $1,000^{\mathrm{F}}$ & $1,000^{\mathrm{F}}$ & $0,483^{\mathrm{F}}$ & $0,853^{\mathrm{F}}$ & 0,209 & 0,247 & 0,395 & 0,261 & $1,000^{\mathrm{F}}$ & $0,238^{\mathrm{F}}$ & $1,000^{\mathrm{F}}$ & $0,357^{\mathrm{F}}$ \\
\hline Q7 & 0,803 & 0,384 & 0,804 & 0,037 & 0,277 & 0,305 & 0,709 & 0,199 & 0,861 & 0,259 & 0,993 & 0,551 \\
\hline Q8 & 0,465 & & & & 0,005 & & 0,001 & 0,001 & & & & 0,033 \\
\hline Q9 & $1,000^{\mathrm{F}}$ & $1,000^{\mathrm{F}}$ & $0,510^{\mathrm{F}}$ & $0,232^{\mathrm{F}}$ & 0,078 & & & & & & & \\
\hline Q10 & 0,184 & 0,169 & 0,869 & 0,309 & 0,296 & & 0,741 & 0,346 & & 0,765 & 0,710 & 0,039 \\
\hline Q11 & 0,696 & 0,702 & 0,372 & 0,398 & 0,703 & 0,571 & 0,601 & 0,570 & 0,370 & 0,736 & 0,518 & 0,874 \\
\hline Q12 & 0,953 & $0,086^{\mathrm{F}}$ & 0,525 & 0,340 & 0,684 & 0,547 & 0,406 & 0,518 & 0,264 & 0,203 & 0,370 & 0,721 \\
\hline Q14 & 0,806 & 0,317 & 0,237 & 0,299 & 0,934 & 0,932 & 0,653 & 0,950 & 0,261 & 0,953 & 0,285 & 0,649 \\
\hline Q17 & $0,680^{\mathrm{F}}$ & $0,708^{\mathrm{F}}$ & $0,539^{\mathrm{F}}$ & $0,322^{\mathrm{F}}$ & 0,479 & 0,267 & 0,151 & 0,273 & 0,440 & 0,139 & 0,257 & $0,675^{\mathrm{F}}$ \\
\hline Q18 & 0,248 & $0,473^{\mathrm{F}}$ & $0,879^{\mathrm{F}}$ & $0,501^{\mathrm{F}}$ & 0,066 & 0,020 & 0,026 & 0,022 & $0,718^{\mathrm{F}}$ & $0,065^{\mathrm{F}}$ & 0,093 & 0,274 \\
\hline Q20 & 0,792 & 0,979 & 0,330 & $<\mathbf{0 , 0 0 1}$ & 0,895 & 0,730 & 0,253 & 0,715 & 0,626 & 0,427 & 0,817 & 0,812 \\
\hline
\end{tabular}

Legenda: CV = Ciclo de Vida; CS = Condição Sistêmica; SO = Tipo de Serviço Odontológico; FST = Índice de Dentes Funcionais; ICO = Índice de Cuidados Odontológicos; P = Dentes Perdidos; UPP = Uso de Prótese Parcial; UPT = Uso de Prótese Total; NPP = Necessidade de Prótese Parcial; NPT $=$ Necessidade de Prótese Total; $\mathrm{Q}^{\mathrm{n}}=$ Questão n. ${ }^{\mathrm{Q}}$ Valor obtido através do Teste do Qui quadrado. ${ }^{\mathrm{F}} \mathrm{O}$ Teste Exato de Fisher foi aplicado quando não foi possível a utilização do Qui quadrado. ${ }^{\mathrm{M}}$ Valor obtido através do Teste U de Mann-Whitney. Em negrito: estatística significante à $<0,05$.

cação e obtenção de vínculo com o profissional entre os adultos e idosos hipertensos e diabéticos.

Apesar de Políticas Públicas de Saúde priorizarem portadores de $\mathrm{DCNT}^{13}$ na Atenção à Saúde, observa-se em relação à Saúde Bucal condições semelhantemente precárias de hipertensos e diabéticos quando esses são comparados à populações sem as referidas DCNT ${ }^{24}$. Dessa forma, os Serviços de Saúde Bucal, sejam públicos ou privados, não têm conseguido prover um cuidado diferenciado a essa população.

\section{Saúde bucal, utilização e percepção sobre os serviços odontológicos}

A amostra de respondentes compôs-se, predominantemente, por mulheres e idoso(a)s, sujeitos que com frequência são os mais encontrados em levantamentos domiciliares ${ }^{23}$. Em relação à condição sistêmica, predominam os que apresentam apenas HAS.

Embora esta pesquisa tenha sido realizada em áreas adscritas à ESF contendo ESB, pouco mais de um quinto dos entrevistados elegeram a ESF como sua referência para tratamentos odontológicos, sendo que quase metade dos entrevistados utiliza o serviço privado. Dessa forma, a aplicação do ASBAP-usuário, inicialmente escolhido justamente por ter sido desenhado na lógica da ESF, teve que ser reformatada para o alcance dos objetivos do estudo. A supressão e até mesmo a alteração de questões se deu para que o instrumento pudesse também avaliar a percepção dos usuários das Clínicas de Ensino e do Serviço Privado.

Destacável é o fato de dois sujeitos relatarem confeccionar suas próteses diretamente com profissionais de nível médio (Gráfico 1), não habilitados legal e tecnicamente no desempenho de tal atividade. O exercício ilegal da Odontologia, além de oferecer riscos sanitários e sociais à população, desguarnecendo-a, principalmente, em qualidade técnica, científica e humanística, corrobora com a desregulação de um setor de serviços que, em última análise, visa produzir saúde e bem-estar - atributos inalcançáveis nos referidos moldes ${ }^{25}$. Outros dois sujeitos não se submetem a acompanhamentos odontológicos há pelo menos dez anos. Mesmo moradores de áreas em que o direito à Atenção à Saúde Bucal materializa-se, a falta de acesso aos serviços, seja por opção própria ou por barreiras impostas por condições socioculturais, ainda fere consideráveis frações da população com a naturalização da perda dentária ${ }^{26,27}$ e consequente sequestro de valores como dignidade e cidadania. 
Em relação às CCSB, observa-se elevado CPOD, baixos FS-T e ICO e preocupante edentulismo, o que não difere, porém, de populações com características semelhantes ${ }^{23,28}$. Como consequência da vasta perda dental, é também elevado o uso de próteses, sobretudo as dentaduras totais, sendo ainda consideráveis as necessidades, em especial, por próteses parciais (Tabela 1). A população hoje adulta e idosa sofre as consequências de condições que vivenciou em um processo acumulativo de riscos durante o ciclo vital $^{17}$, somado à - num passado recente - ausência de políticas públicas focadas em promoção e prevenção e presença de modelos de Atenção voltados ao mercado, à mutilação e ao artificialismo protesista $^{26,27}$.

Apesar de precárias CCSB - elevados edentulismo e demanda por próteses -, os adultos e idosos entrevistados avaliaram positivamente os serviços, de maneira geral, observando-se altas frequências de respostas que exprimem satisfação, com exceção da questão 8 (que refere-se ao vínculo/longitudinalidade do cuidado), onde as opiniões parecem estar mais divididas (Tabela 1). Em relação aos usuários das Clínicas de Ensino e das ESF, poderia se imaginar que um possível receio de se perder o acesso aos tratamentos restringiria sua capacidade de apontar eventuais falhas nos serviços. Entre os usuários do Serviço Privado, contudo, o caráter positivo das avaliações deve realmente apontar para um horizonte de significativa satisfação.

\section{Determinantes da percepção sobre serviços odontológicos}

A Percepção dos entrevistados sobre os Serviços Odontológicos parece não ser influenciada por Sexo, Ciclo de Vida (Adulto/Idoso) e Condição Sistêmica (HAS/DM) em nenhum dos itens avaliados pelo ASBAP-usuário $(\rho>0,05)$ (Tabela 2).

Entretanto, ao se comparar os três diferentes Tipos de Serviços Odontológicos, algumas diferenças emergem. De maneira geral, pode-se inferir que os usuários das Clínicas de Ensino estão mais satisfeitos com a comunicação estabelecida com os profissionais que os frequentadores de outros serviços. Por outro lado, entre esses sujeitos é maior o número dos que consideram o serviço lento, de difícil acesso, estabelecendo-se pouco vínculo (o dentista não conhece bem os usuários). Por ser um Serviço, onde muitas vezes o objetivo principal é o aprendizado de graduandos, tais aspectos são compreensíveis. Entretanto, o que não deveria ser relevado é o fato de que, em determinadas especialidades, esses pontos mostram-se como a única alternativa 'gratuita' (leia-se: pública) de acesso. Dessa forma, é mister que a Rede de Atenção à Saúde Bucal contemple a integralidade do cuidado em todos os níveis, não restringindo-se apenas à Atenção Primária ${ }^{29}$.

Ao Serviço Privado atribuiu-se maior vínculo e facilidade de acesso, o que não causa estranheza. Pois, a parcela da população que consegue pagar diretamente ou por arranjos mutualistas por serviços de saúde, geralmente, acessam esses em condição diferenciada. Nesses casos, há ainda, por parte dos prestadores do serviço, uma preocupação em estender e perpetuar laços com seus clientes $^{30}$.

Já na ESF, o Serviço foi considerado mais rápido. Porém, características como acesso, vínculo/ longitudinalidade e comunicação ainda deixam a desejar. Tal resultado preocupa, tendo em vista que essas dimensões integram os atributos essenciais da Atenção Primária à Saúde (APS) ${ }^{29}$. Não encontrando tais qualidades num Serviço Público, o sujeito em condição socioeconômica favorável busca o Serviço Privado. Entretanto, há de se considerar que o acesso diferenciado a serviços expressa uma clara e legítima injustiça social.

Aliás, o vínculo/longitudinalidade - avaliado especificamente pela questão 8 - relaciona-se não apenas ao tipo de serviço, mas também às CCSB. Observa-se menor número de dentes perdidos, maior número de dentes funcionais (FS-T) e cuidado mais conservador (maior ICO) nos sujeitos que acreditam que o dentista os conheça bem. Um interessante paralelo pode ser estabelecido, confrontando-se essas CCSB com a avaliação da estrutura tecnológica do Serviço (questão 18). Os sujeitos que consideram os equipamentos utilizados pelo dentista como bons e em adequado funcionamento - ao contrário do que se poderia imaginar - apresentam maior número de dentes perdidos e menores números de dentes funcionais e ICO. Diante de maior densidade tecnológica, a perda dental pode ser apenas uma etapa para a confecção de próteses que simbolizem sofisticação e avanço tecnológico, como possivelmente ocorre no Serviço Privado. Tal achado aponta, ainda, para a necessidade de se consolidar a oferta de serviços com orientação humanizada, considerando-se a comunidade como a interação de sujeitos biopsicossociais, na perspectiva da determinação social ${ }^{31}$, uma vez que $o$ modelo biomédico, calcado no tecnicismo, evidencia graves fragilidades e insuficiências na produção de saúde. 
A relação entre a Percepção sobre os Serviços Odontológicos e o uso/necessidade de próteses coloca a Prótese Total como o símbolo de desvinculação do usuário para com seu serviço (relação entre questão 8 e uso/necessidade de PT, Tabela 2). Reforçando esses achados, observa-se que aqueles que necessitam de PT são também os que mais julgam ser difícil o acesso aos serviços (questão 1), além de serem os que mais consideram que o dentista não os conhece bem (questão 8), nem se preocupa com sua saúde (questão 10). Sendo a Prótese Total o estágio final de um ciclo de dor, sofrimento e mutilações ${ }^{26,27}$, o contato com o profissional tende a diminuir - ocorrendo sazonalmente para manutenção ou troca das próteses - ou até mesmo se extinguir - considerando-se o uso da mesma prótese por mais de dez anos por alguns sujeitos (Gráfico 1). Assim, o distanciamento entre serviço e usuário tornase algo quase inevitável. Sendo esse também um fator complicador ao acesso às próteses - geralmente um nó crítico pelo custo que representam seja no setor público ou na iniciativa privada.

\section{Limitações do estudo}

Aponta-se como limitações deste estudo seu corte transversal, o que não permite inferências causais, além da alteração do instrumento (ASBAP-usuário) utilizado para verificar a Percepção sobre os Serviços Odontológicos na amostra estudada. A supressão de quatro perguntas do questionário original, tendo em vista a adequação ao universo de sujeitos (oriundos de serviços diversos de Saúde Bucal além das ESF) pode ter influenciado em algum grau na obtenção dos resultados, exigindo cautela na análise dos dados.

\section{Conclusões}

A avaliação de serviços de saúde na perspectiva do usuário corrobora para a concretização da participação popular no Sistema Único de Saúde. Pois, assim, reconhece-se a centralidade desse ator e promove-se o 'empoderamento' de indivíduos e coletividades, a partir da criação de um ambiente virtuoso em que o próprio processo avaliativo expressa o fortalecimento das capacidades de ação, da criação de oportunidades de reflexões e aprendizagens ${ }^{4}$ - inclusive em relação à autoprodução de saúde.

Na população estudada a Saúde Bucal é melhor entre os sujeitos que relatam bom vínculo com o dentista e pior entre os que consideram bons os equipamentos utilizados nos Serviços.

\section{Colaboradores}

Os autores EJP Oliveira, DA Nogueira, AA Pereira trabalharam em todas as etapas de elaboração do artigo. 


\section{Referências}

1. Donabedian A. Enfoques básicos para la evaluación. In: La qualidad de la atencion médica: definición e métodos de evaluación. Cidade do México: La Prensa Médica Mexicana; 1984. p. 97-157.

2. Favaro P, Ferris LE. Program evaluation with limited fiscal and human resources. Cad Saude Publica 1991; 11(3):425-438.

3. Ware Junior JE, Snyder MK, Wright WR, Davies AR. Defining and measuring patient satisfaction with medical care. Eval program plan 1983; 6(3):247-263.

4. Kleba ME, Wendhausen ALP. O processo de pesquisa como espaço e processo de empoderamento. Interface (Botucatu) 2010; 14(33):427-436.

5. Shi L, Starfield B, Xu J. Validating the adult primary care assessment tool. J Fam Practice 2001; 50(2):161171.

6. Almeida C, Macinko J. Validação de uma metodologia de avaliação rápida das características organizacionais e do desempenho dos serviços de atenção básica do Sistema único de Saúde (SUS) em nível local. Brasil: OPAS/Ministério da Saúde; 2006.

7. Fernances LMAG. Validação de um instrumento para avaliação da satisfação dos usuários com os serviços públicos de saúde bucal: QASSaB [tese]. Camaragibe: Universidade Federal de Pernambuco; 2002.

8. Sanchez HF. Construção e validação de um instrumento para avaliação dos serviços públicos de saúde bucal na atenção primária à saúde sob a ótica dos usuários (AS$B A P$-usuário) [tese]. Belo Horizonte: Universidade Federal de Minas Gerais; 2013.

9. Fadel MAV, Filho GIR. Percepção da qualidade em serviços públicos de saúde: um estudo de caso. Revista de Administração Pública 2009; 43(1):7-22.

10. Mendes VLPS. Avaliação dos serviços de saúde por usuários: questão de cidadania. Revista Baiana de Enfermagem 2003; 18(1):97-110.

11. Costa APS, Machado FCA, Pereira ALBP, Carreiro AFP, Ferreira MAF. Qualidade técnica e satisfação relacionadas às próteses totais. Cien Saude Colet 2013; 18(2):453-460.

12. Mendes EV. As redes de atenção à saúde. Cien Saude Colet 2010; 15(5):2297-2305.

13. Brasil. Ministério da Saúde (MS). Plano de Ações Estratégicas para o Enfrentamento das Doenças Crônicas Não Transmissíveis (DCNT) no Brasil-2011-2022. Brasília: MS; 2011.

14. Chávarry NG, Vettore MV, Sansone C, Sheiham A. The relationship between diabetes mellitus and destructive periodontal disease: a meta-analysis. Oral health prev dent 2009; 7(1):107-127.

15. Herring ME, Shah SK. Periodontal Disease and Control of Diabetes Mellitus. JAOA 2006; 106(1):416-421.

16. Salvi GE, Carollo-Bittel B, Lang NP. Effects of diabetes mellitus on periodontal and peri-implant conditions: update on associations and risks. $J$ clin periodontal 2008; 35(8):389-409.

17. Peres MA, Peres KG. A saúde bucal no ciclo vital: acúmulo de riscos ao longo da vida. In: Antunes JLF, Peres MA. Epidemiologia da saúde bucal. Rio de Janeiro: Guanabara Kogan; 2006. p. 249-259.
18. World Health Organization (WHO). Oral health surveys: basic methods. $5^{\text {th }}$ ed. Geneva: ORH/EPID; 2013.

19. Sheiham A, Maizels J, Maizels A. New composite indicators of dental health. Community dent health 1987; 4(4):407-414

20. Walsh J. International patterns of oral health care: the example of New Zealand. N Z Dent J 1970; 66(1):143152.

21. Brasil. Ministério da Saúde (MS). SB-BRASIL 2010 Pesquisa Nacional de Saúde Bucal: Proposta de Projeto Técnico para Consulta Pública. Brasília: Ministério da Saúde; 2009.

22. Silva NN. Amostragem probabilística. São Paulo: EDUSP; 1998.

23. Brasil. Ministério da Saúde (MS). SB-BRASIL 2010 Pesquisa Nacional de Saúde Bucal: Principais Resultados. Brasília: MS; 2011.

24. Moreira RS, Nico LS, Tomita NE. O risco espacial e fatores associados ao edentulismo em idosos em município do sudeste do Brasil. Cad Saude Publica 2011; 27(10):2041-2053.

25. Carvalho CL. Dentistas práticos no Brasil: história de exclusão e resistência na profissionalização da odontologia brasileira [tese]. Rio de Janeiro: Fiocruz; 2003.

26. Oliveira AGRC. Edentulismo. In: Antunes JLF, Peres MA. Epidemiologia da saúde bucal. Rio de Janeiro: Guanabara Kogan; 2006. p. 205-218.

27. Souza ECF. Formação e trabalho em Odontologia: ampliar a clínica para construir uma nova cultura de cuidado em saúde bucal. Texto para subsidiar a III Conferência Estadual de Saúde Bucal do RN. Natal: SES/ $\mathrm{RN} ; 2004$.

28. Miotto MH, Barcellos LA, Veltren DB. Avaliação do impacto na qualidade de vida causado por problemas bucais na população adulta e idosa em município da região sudeste. Cien Saude Colet 2012; 17(2):397-406.

29. Starfield B. Atenção primária: equilíbrio entre necessidades de saúde, serviços e tecnologia. Brasília: UNESCO; 2002.

30. Castanheira CHC, Pimenta AM, Lana FCF, Malta DC. Utilização de serviços públicos e privados de saúde pela população de Belo Horizonte. Rev Bras Epidemiol 2014; 17(Supl. 1):256-266.

31. Dahlgren G, Whitehead M. Policies and strategies to promote social equity in health. Arbetsrapport: Institute for Future Studies; 1991.

Artigo apresentado em 15/02/2016

Aprovado em 20/10/2016

Versão final apresentada em 22/10/2016 